\title{
Plomo sanguíneo en los habitantes de cuatro localidades peruanas
}

\author{
Augusto V. Ramírez, ${ }^{1}$ Juan Cam Paucar ${ }^{2}$ y José M. Medina ${ }^{3}$
}

RESUMEN Con objeto de conocer las concentraciones de plomo sanguíneo de los habitantes de ciudades peruanas de diferente densidad poblacional y grado de desarrollo industrial, durante 1994 y 1995 se efectuó un estudio transversal con una muestra aleatoria de 180 hombres y 180 mujeres sin exposición ocupacional al plomo y procedentes de Lima, Huancayo, La Oroya y Yaupi. Se midió el plomo sanguíneo por el método de absorción atómica con espectrofotómetro Perkin Elmer 603 sin horno de grafito.

Los resultados revelaron concentraciones de plomo en sangre de $269 \pm 63 \mu \mathrm{g}$ por litro $(\mu \mathrm{g} / \mathrm{L})$ en Lima, $224 \pm 47 \mu \mathrm{g} / \mathrm{L}$ en Huancayo; $348 \pm 40 \mu \mathrm{g} / \mathrm{L}$ en La Oroya, y $140 \pm$ $27 \mu \mathrm{g} / \mathrm{L}$ en Yaupi. Se saca la conclusión de que las concentraciones de plomo sanguíneo de los habitantes de estas ciudades guardan relación con el grado de industrialización y la densidad poblacional de cada localidad.

En las últimas décadas, el aire de las capitales de casi todos los países latinoamericanos se ha deteriorado. Ello ha sido consecuencia de la instalación de industrias en su periferia - e incluso en centros urbanos- y del rápido aumento de su obsoleto parque automotor, que utiliza combustible fósil con aditivos de plomo (1). Esta circunstancia empeora cuando, como en el caso de Lima, Perú, la ciudad no cuenta con ningún sistema de transporte público.

En el Perú, la migración no planificada del campo a la ciudad agrava ese

\footnotetext{
1 Departamento de Salud Ocupacional Centromin Perú, S.A. Las solicitudes de separatas deben enviarse a este autor a la siguiente dirección postal: Las Mandarinas 210-306, Lima 12, Perú.

2 Instituto Nacional de Ciencias Neurológicas, Lima, Perú.

3 Servicio urbano marginal de salud, Unidad Básica de Salud, Junín, Perú.
}

problema. El aumento consiguiente de la densidad poblacional aumenta la necesidad de disponer de servicios de transporte y trae consigo la aparición de pequeñas industrias caseras, como fábricas clandestinas de acumuladores (baterías), soldadores de piezas de automóviles en la vía pública, comercios ambulatorios, y demás.

En el interior del Perú, las pequeñas ciudades han comenzado a padecer estos mismos problemas desde que las villas se han convertido en ciudades semiindustriales. La Oroya, por ejemplo, es una ciudad industrial con un alto nivel de contaminación atmosférica (2).

Aunque la contaminación atmosférica de Lima es palpable (3), no se han publicado estudios sobre la contaminación de esta ciudad por plomo ambiental. Los vientos transportan los contaminantes atmosféricos a grandes distancias de su fuente de origen e incluso a zonas rurales presuntamente no contaminadas (4-5). Uno de estos contaminantes es el plomo, metal pesado que es tóxico para el hombre y para los ecosistemas. Su concentración en la sangre de los habitantes de las ciudades se puede conocer y constituye un buen indicador del plomo ambiental (6-12).

El objetivo de este trabajo es conocer la concentración de plomo en la sangre $(\mathrm{Pb}-\mathrm{S})$ de los habitantes de ciudades peruanas con diferente densidad poblacional y grado de desarrollo industrial. Asimismo, se analiza la distribución de la concentración de $\mathrm{Pb}-\mathrm{S}$ de los habitantes de ciudades peruanas con distinta densidad de población, que no están expuestos a este metal durante su desempeño ocupacional. No se pretende estimar valores de tendencia central de esa distribu- 
ción ni cifras de normalidad, habida cuenta del pequeño tamaño de las muestras del estudio. La carencia de recursos impide realizar muestreos más amplios. Por este motivo, la presente investigación se diseñó como un estudio piloto destinado a aportar datos preliminares útiles para realizar estudios epidemiológicos en el futuro.

\section{MATERIALES Y MÉTODOS}

En cada una de las ciudades donde se llevó a cabo el estudio se estudiaron 80 personas, 40 de cada sexo, todas ellas de edades comprendidas entre los 18 y 50 años y residentes permanentes en la ciudad. En La Oroya y Huancayo la muestra de 80 personas se integró con voluntarios reclutados en la calle. A estas personas se les explicó el objetivo del estudio después de haber hecho una campaña de información en la ciudad. En Lima, los 80 voluntarios se reclutaron entre los acompañantes de pacientes que acudían al Instituto de Neurología. En Yaupi, una pequeña aldea, la muestra de 80 voluntarios constituye gran parte de la población adulta.

El diseño de esta investigación fue transversal (13). En el estudio se incluyeron cuatro localidades. Para seleccionarlas, además de los criterios de inclusión mencionados anteriormente, se tuvieron en cuenta sus características demográficas contrastables y el grado de industrialización (14). Se excluyó a las personas que hubiesen podido estar expuestas al plomo orgánico o inorgánico. Las localidades participantes fueron las siguientes:

- Lima, capital del Perú. Su población asciende a 7 millones de habitantes $(30 \%$ de la población peruana) y la densidad poblacional es de 184 habitantes por $\mathrm{km}^{2}$. La ciudad, situada a $120 \mathrm{~m}$ sobre el nivel del mar, padece todos los problemas de la capital de un país en desarrollo: sobrepoblación, inmigración caótica, enorme parque automotor obsoleto que utiliza combustibles fósiles con anti- detonante a base de plomo, carencia de vehículos de transporte colectivo, etc. $(1,3,14)$.

- Huancayo, ciudad de la sierra central. Se sitúa a $3240 \mathrm{~m}$ sobre el nivel del mar y tiene 300000 habitantes y una densidad poblacional de 55 habitantes por $\mathrm{km}^{2}$. Es el centro comercial e industrial más importante de la sierra central peruana y ha experimentado un rápido crecimiento $\mathrm{y}$ presenta problemas similares a los de Lima aunque en menor escala (14).

- La Oroya, ciudad metalúrgica. Está situada a $3730 \mathrm{~m}$ sobre el nivel del mar. Su población es de 40000 habitantes y su densidad poblacional de 100 habitantes por $\mathrm{km}^{2}$. La ciudad padece problemas notorios de contaminación ambiental de origen industrial (2).

- Yaupi, aldea de ceja de selva completamente rural. Su población es de 300 habitantes. La aldea carece, prácticamente, de parque automotor. La principal ocupación de sus habitantes es la agricultura no mecanizada.

Para medir la concentración de $\mathrm{Pb}-\mathrm{S}$, se extrajeron $8 \mathrm{~mL}$ de sangre venosa a cada uno de los participantes con una jeringa de vidrio heparinizada, y previamente lavada con ácido nítrico diluido. Las muestras obtenidas se sellaron y refrigeraron hasta el momento de analizarlas. A continuación se midió la concentración de plomo por absorción atómica, después de realizar la extracción con metilisobutilcetona, con un espectrofotómetro Perkin Elmer 603 sin horno de grafito (15). Este aparato es calibrado periódicamente por sus operadores y por auditores externos.

El análisis estadístico de los datos consistió en el cálculo de la media aritmética $(\bar{X})$ de la concentración de $\mathrm{Pb}-\mathrm{S}$ y su desviación estándar (DE), así como de la media de la edad de los integrantes de cada grupo. Para comparar las medias de la concentración de $\mathrm{Pb}-\mathrm{S}$ de cada grupo y las de ambos sexos, se utilizó la $t$ de Student a un nivel de significación estadística de 5\% (16). Para hacer las comparaciones, el grupo IV se utilizó como grupo control.

\section{RESULTADOS}

En el cuadro 1 aparecen la media y la $\mathrm{DE}$ de las concentraciones de $\mathrm{Pb}-\mathrm{S}$ y de la edad por sexo y localidades. La media de la concentración de $\mathrm{Pb}-\mathrm{S}$ varió en razón del tamaño y las características de las localidades participantes. Esta media fue más elevada en Lima que en Huancayo y menor en Yaupi. En los habitantes de La Oroya se registraron las mayores medias de la concentración de $\mathrm{Pb}-\mathrm{S}$.

En el cuadro 2 se presentan los resultados de las comparaciones entre las medias de la concentración de $\mathrm{Pb}-\mathrm{S}$ en las cuatro localidades. La comparación muestra que las diferencias estadísticamente significativas fueron las observadas entre los grupos I y IV, II y IV, y III y IV. Por otra parte, la media de la concentración de $\mathrm{Pb}-\mathrm{S}$ en los hombres fue de 257,5 $(n=160 ; \mathrm{DE}=45) \mathrm{y}$ en las mujeres de 233,3 ( $n=160 ; \mathrm{DE}=43)$. Las diferencias entre ambas medias no fueron estadísticamente significativas $(t=$ 0,9812; $P<0,5)$.

\section{DISCUSIÓN}

El $80 \%$ del plomo del aire atmosférico, $90 \%$ del cual se encuentra en forma de partículas inorgánicas, proviene de la gasolina (17). Las principales fuentes de emisión de plomo a los ecosistemas son las fundiciones de plomo $(2,4,7)$ y las industrias clandestinas de acumuladores (6). El agua potable puede contaminarse en su misma fuente por el plomo del aire o por vertidos de la escoria de las minas que no se procesan. La OMS ha fijado una concentración máxima tolerable de plomo en el agua potable de 0,05 ppm (18).

Otra vía de ingreso del plomo en el cuerpo humano es la ingestión de alimentos contaminandos, tales como verduras, frutas y tubérculos cultivados con agua de regadío contaminada. La leche de vaca y la materna también son 
CUADRO 1. Concentraciones de plomo sanguíneo (Pb-S) por grupo y sexo en las cuatro localidades estudiadas. Lima, Perú, 1994-1995

\begin{tabular}{|c|c|c|c|c|c|c|c|c|c|}
\hline Grupo & $n$ & Sexo ${ }^{a}$ & $\begin{array}{l}\bar{X}_{\mathrm{Pb}-\mathrm{S}} \\
(\mu \mathrm{g} / \mathrm{L})\end{array}$ & $\begin{array}{l}\mathrm{DEM}^{\mathrm{b}} \\
(\mu \mathrm{g} / \mathrm{L})\end{array}$ & $\begin{array}{c}\mathrm{VE}^{\mathrm{c}} \\
(\mu \mathrm{g} / \mathrm{L})\end{array}$ & $\begin{array}{c}\bar{X}_{\text {Edad }} \\
\text { (añ̃os) }\end{array}$ & $\begin{array}{c}\mathrm{ME}^{\mathrm{d}} \\
\text { (años) }\end{array}$ & $\begin{array}{l}\text { Habitantes } \\
(\times 1000)\end{array}$ & $\mathrm{DP}^{\mathrm{e}}$ \\
\hline Grupo I & 40 & $\mathrm{H}$ & 277 & 54 & & 29,3 & 29 & 7000 & 184 \\
\hline Lima & 40 & M & 261 & 72 & & 23,5 & 24 & & \\
\hline Total & 80 & & 269 & 63 & $170-350$ & 26,4 & & & \\
\hline Coeficiente de variación (\%) & 23 & & & & & & & & \\
\hline Grupo II & 40 & $\mathrm{H}$ & 237 & 46 & & 26,3 & 25 & 300 & 55 \\
\hline Huancayo & 40 & M & 211 & 47 & & 24,9 & 25 & & \\
\hline Total & 80 & & 224 & 47 & $150-280$ & 25,6 & 25 & & \\
\hline Coeficiente de variación (\%) & 20 & & & & & & & & \\
\hline Grupo III & 40 & $\mathrm{H}$ & 365 & 51 & & 23,4 & 22 & 50 & 100 \\
\hline La Oroya & 40 & $M$ & 331 & 30 & & 23,0 & 22 & & \\
\hline Total & 80 & & 348 & 40 & $280-400$ & 23,0 & 22 & & \\
\hline Coeficiente de variación (\%) & 11 & & & & & & & & \\
\hline Grupo IV & 40 & $\mathrm{H}$ & 151 & 30 & & 26,7 & 26 & 0.3 & $\ldots$ \\
\hline Yaupi & 40 & M & 130 & 23 & & 23,3 & 25 & & \\
\hline Total & 80 & & 140 & 27 & $80-170$ & 25,0 & 26 & & \\
\hline Coeficiente de variación (\%) & 19 & & & & & & & & \\
\hline
\end{tabular}

a $\mathrm{H}=$ hombre; $\mathrm{M}=$ mujer.

b DEM = Desviación estándar de la media.

c VE $=$ Valores extremos.

$\mathrm{d} M E=$ mediana de edad del grupo.

e DP = densidad poblacional (habitantes por $\mathrm{km}^{2}$ ).

fuentes de ingestión de plomo, ya que en ellas se ha detectado plomo al igual que en los alimentos enlatados (6).

La absorción de plomo por vía respiratoria depende de su concentración en el ambiente, así como del tiempo y la frecuencia de la exposición. En el organismo, el plomo es transportado por la sangre (18) y distribuido a los diferentes órganos, en algunos de los cuales se acumula. Si la exposición es continua, se alcanza un equilibrio dinámico en los diferentes compartimientos del organismo. En la sangre, 90\% del plomo se encuentra en los eritrocitos (18-22), se almacena en los huesos y se elimina principalmente por la orina $y$ por el cabello y las uñas (18). Durante

CUADRO 2. Resultados de las comparaciones de la concentración de plomo sanguíneo de las cuatro localidades estudiadas. Lima, Perú, 1994-1995

\begin{tabular}{ccc}
\hline $\begin{array}{c}\text { Grupos } \\
\text { de comparación }\end{array}$ & $t$ de Student & $P$ \\
\hline I y IV & 2,015 & $<0,02$ \\
II y IV & 1,895 & $<0,02$ \\
III y IV & 2,492 & $<0,02$ \\
\hline
\end{tabular}

este recorrido, el plomo produce efectos tóxicos y alteraciones enzimáticas, que se utilizan como indicadores biológicos. Entre ellos destacan la actividad de la deshidratasa del ácido deltaaminolevulínico en la sangre, protoporfirinas de los eritrocitos circulantes, ácido deltaaminolevulínico en la orina y coproporfirinas urinarias (23). Sin embargo, para medir la exposición "actual" a este metal, el $\mathrm{Pb}-\mathrm{S}$ sigue siendo el mejor indicador $(11,21-26)$.

En muchas investigaciones se ha demostrado la presencia creciente del plomo ambiental en las grandes ciudades y en zonas completamente rurales $(4,5)$. Sus valores descienden a medida que disminuye la densidad poblacional. El carácter industrial de la ciudad desempeña un papel preponderante en el nivel de plomo ambiental, que a su vez se correlaciona directamente con la concentración de $\mathrm{Pb}-\mathrm{S}$ de sus habitantes. Se han encontrado valores entre 200 y $400 \mu \mathrm{g} / \mathrm{L}$ en habitantes de grandes ciudades o de ciudades industriales, mientras que en zonas rurales este valor oscila en alrededor de 120 $\mu \mathrm{g} / \mathrm{L}$. También se han detectado diferencias entre los dos sexos: la concen- tración de $\mathrm{Pb}-\mathrm{S}$ es más alta en hombres que en mujeres $(6,12,17,18,20,21,24)$.

La concentración de $\mathrm{Pb}-\mathrm{S}$ que actualmente se considera el límite de la normalidad es $\leq 1,4 \mu \mathrm{mol} / \mathrm{L}(18,22)$. Algunos investigadores escandinavos han demostrado que a esta concentración se producen alteraciones subclínicas que pueden detectarse en los análisis de la sangre. Las personas con estas concentraciones se encuentran en riesgo de desarrollar cáncer cerebral o pulmonar. Por este motivo, dicho límite se ha reducido a 0,7 $\mu \mathrm{mol} / \mathrm{L}$ (145 $\mu \mathrm{g} / \mathrm{L})(9,11,27)$. Sin embargo, las concentraciones de $\mathrm{Pb}-\mathrm{S}$ encontradas en grupos seleccionados de habitantes de grandes ciudades siempre han sido elevadas (por ejemplo, 1,44 $\mu \mathrm{mol} / \mathrm{L}$ en México y 0,87 $\mu \mathrm{mol} / \mathrm{L}$ en Bélgica) (24).

Los resultados obtenidos en los grupos de Huancayo (224 $\pm 47 \mu \mathrm{g} / \mathrm{L})$, Lima (269 $\pm 63 \mu \mathrm{g} / \mathrm{L})$ y La Oroya (348 $\pm 40 \mu \mathrm{g} / \mathrm{L}$ ) concuerdan con los de estudios realizados en ciudades similares (19-21, 24). Estos hallazgos son atribuibles a la exposición de estas personas a las concentraciones elevadas de plomo del ambiente. En el grupo de Yaupi, que sirvió de grupo control en 
este estudio, la concentración media de $\mathrm{Pb}-\mathrm{S}$ fue de $140 \pm 27 \mu \mathrm{g} / \mathrm{L}$, cifra ligeramente mayor que las detectadas en zonas rurales por otros autores (5), aunque se encuentra dentro de los límites de la normalidad aceptados por investigadores escandinavos (9). Esta concentración puede explicarse por la ingestión del plomo que contienen los alimentos enlatados.

Finalmente, se encontraron diferencias estadísticamente significativas entre las medias de la concentración de $\mathrm{Pb}-\mathrm{S}$ de los grupos de Lima, Huancayo y La Oroya, y las del grupo de
Yaupi. Por el contrario, no se detectaron diferencias estadísticamente significativas entre las medias de la concentración de $\mathrm{Pb}-\mathrm{S}$ de ambos sexos.

Las concentraciones de $\mathrm{Pb}-\mathrm{S}$ de los habitantes de las ciudades estudiadas se relacionan directamente con el grado de industrialización y la densidad poblacional de la localidad. La media más baja de la concentración sanguínea de plomo se registró en los habitantes de Yaupi $(140 \mu \mathrm{g} / \mathrm{L})$ y la más alta, en los de La Oroya (348 $\mu \mathrm{g} / \mathrm{L}$ ). Las concentraciones sanguíneas de $\mathrm{Pb}$-S detectadas en los habitan- tes de Lima, La Oroya y Huancayo se consideran excesivas.

En el estudio de la contaminación ambiental de las ciudades debería incluirse la medición de la concentración de $\mathrm{Pb}$-S, además de los consabidos óxidos de nitrógeno y azufre, ozono, monóxido de carbono y material en partículas.

Por consiguiente, se concluye que el $\mathrm{Pb}-\mathrm{S}$ de los habitantes de las localidades peruanas estudiadas es directamente proporcional al tamaño de la localidad y a su grado de desarrollo industrial.

\section{REFERENCIAS}

1. Weitzenfeld H. Contaminación atmosférica y salud en América Latina. Bol Oficina Sanit Panam 1992;112:97-109.

2. Alarcón AC. Los humos de La Oroya. En: Instituto para el Desarrollo de la Pesca y la Minería. Taller sobre el medio ambiente: La Oroya 1994. Lima: IPEMIN; 1994.

3. Diario El Comercio. "Contaminación ambiental". Lima, domingo 16 de abril de 1995:A-12, A-13.

4. Jernigan EL, Ray BS, Duce RA. Lead and bromine in atmospheric particulate matter on Oahu, Hawaii. Atmos Environ 1971;5:881-886.

5. Murozumi MT, Chow J, Patterson CC. Chemical concentration of pollutant lead aerosols, terrestrial dust and sea salts in Greenland and Antartic snow strata. Geochem Cosmochem Acta 1969;33:1237-1294.

6. Vega S. Evaluación epidemiológica de riesgos causados por agentes químicos ambientales: cinética y efectos de los contaminantes tóxicos del ambiente. México, DF: Centro Panamericano de Ecología Humana y Salud, Organización Panamericana de la Salud; 1985.

7. Pedlowsky MA. Entradas atmosféricas de nutrientes, metais pesados e acidez livre na Baía de Sepetiva [tesis de maestría]. Rio de Janeiro: Instituto de Geociencias UFRJ; 1991.

8. Organización Mundial de la Salud. Investigación de brotes de enfermedades ambientales: signos, sintomas y sus posibles etiologías ambientales. Ginebra: OMS; 1993. (Manual de entrenamiento OMS.)

9. Anttila A, Heikkilä P, Nykysi E, et al. Risk of nervous system cancer among workers exposed to lead. J Occup Environ Med 1996:38: 131-136.
10. Steenland K, Selevan S, Landrigan P. The mortality of lead smelters workers: an update. Am J Public Health 1992;82:1641-1644.

11. Anttila A, Heikkilä P, Pukkala E, et al. Excess lung cancer among workers exposed to lead. Scand J Work Environ Health 1995;21:460.

12. Silvany-Neto AM, Carvalho F, Tavares T. Evolução da intoxicação por chumbo em crianças de Santo Amaro, Bahia-1980, 1985 e 1992. Bol Oficina Sanit Panam 1996;120:11-22.

13. Reynaga OJ. Epidemiología IV: diseños de investigación para la comprobación de hipótesis epidemiológicas. Estudios transversales y estudios retrospectivos. México, DF: Centro Panamericano de Ecología Humana y Salud, Organización Panamericana de la Salud; 1985.

14. Fondo Editorial Navarrete. Gran atlas universal y del Perú. Lima: Fondo Editorial Navarrete; 1995.

15. Mitchell D, Ryan F, Aldous KM. The precise determination of lead in whole blood by solvent extraction: atomic absortion spectometry. Atomic Absorp Newsletter 1972;2:437-440.

16. Kleinbaum DG, Kupper LL, Morgenstein H. Epidemiologic research principles and quantitative methods. Belmont, Los Angeles: Lifetime Learning Publications; 1982.

17. US Environmental Protection Agency. Air quality criteria for lead. Research Triangle Park North Carolina: EPA; 1983.

18. Organización Panamericana de la Salud. Criterios de salud ambiental 3. Plomo. Washington, DC: OPS; 1979. (Publicación científica 338.)

19. Nogueira DP, et al. Taxa de chumbo em amostra de voluntários "não expostos" habitantes da Grande São Paulo. Rev Bras Saude Ocupacional 1980;30:36-38.
20. Pirkle JL, Brody JB, Gunter EW, et al. The decline in blood lead levels in the United States. JAMA 1994;272:284-291.

21. Landrigan PJ. Epidemic lead absorption near an ore smelter. N Engl J Med 1975;292:123.

22. Organización Mundial de la Salud. Límites de exposición profesional a los metales pesados que se recomiendan por razones de salud. Ginebra: OMS; 1980.

23. Da Silva NR, De Camargo Fonseca ME. Papel dos indicadores biológicos na avaliação da exposição ocupacional ao chumbo. Rev Bras de Saude Ocupacional 1987;58:7-9.

24. Vahter M, ed. Assessment of human exposure to lead and cadmium through biological monitoring. Stockholm: National Swedish Institute of Environment Medicine, Karolinska Institute; 1982.

25. Galvão SA, Gallego Gándara de Fernícola NA, Mendes R. Intoxicação por chumbo. En: Mendes R. Medicina do trabalho. São Paulo: Sarvier; 1980.

26. Levy BS, Wegman DH, eds. Occupational Health. Boston: Little, Brown and Co.; 1988.

27. The National Institute of Occupational Health. The Nordic Expert Group for Criteria Documentation of Health Risk Chemicals. Stockholm: The National Institute of Occupational Health; 1994.

Manuscrito recibido el 26 de mayo de 1996. Aceptado para publicación, tras revisión, el 13 de agosto de 1996. 
ABSTRACT During 1994 and 1995, a cross-sectional study was carried out to investigate the concentrations of lead in the blood of inhabitants of four Peruvian cities (Lima, Huancayo, La Oroya, and Yaupi) with different population densities and degrees of industrial development. In a random sample of 180 men and 180 women without occupational exposure to lead, blood lead levels were measured by the atomic absorption method with a Perkin Elmer 603 spectrophotometer without a graphite oven.

The results revealed blood lead concentrations of $269 \pm 63$ micrograms per liter $(\mu \mathrm{g} / \mathrm{L})$ in Lima, $224 \pm 47(\mu \mathrm{g} / \mathrm{L}$ in Huancayo; $348 \pm 40 \mu \mathrm{g} / \mathrm{L}$ in La Oroya, and $140 \pm$ $27 \mu \mathrm{g} / \mathrm{L}$ in Yaupi. It was concluded that blood lead levels in the inhabitants of these cities were related to the degree of industrialization and the population density of each locality. Peruvian localities

\title{
Conferencia Internacional sobre Salud y Seguridad Ocupacional en el Sector Informal
}

Fechas: 21 a 24 de octubre de 1997

Lugar: Bali

El sector informal es muy importante para la economía de casi todos los países en desarrollo, ya que provee empleo a un número considerable de personas de zonas urbanas y rurales. Sin embargo, por lo general, las personas así empleadas se encuentran en una situación inferior al resto de la población en cuanto a salarios y seguridad social. Esto resulta en mayores riesgos para su salud. Se ha planeado, por lo tanto, esta Conferencia con objeto de proporcionar un foro de discusión e intercambio de experiencias sobre el tema anunciado. Es de esperar que mediante esta experiencia se planteen propuestas practicables de atención primaria para atender de manera más satisfactoria a ese grupo de trabajadores. Los temas seleccionados abarcan los problemas ocupacionales de los agricultores, vendedores ambulantes, trabajadoras domésticas y artesanos, así como el riesgo de contraer sida en el tabajo, la ergonomía en el sector informal, la exposición a sustancias tóxicas y la organización de servicios de salud en el sector informal.

\author{
Información: \\ Secretariat, Bali - Icohis 1997 \\ Occupational Health Unit, DG of Community Health \\ JI. Rasuna Said Block X5, \\ Kav No. 4-9, Floor 7B \\ Jakarta 12950, Indonesia \\ Teléfonos y Fax: 62-21-5221229, 5203871 \\ Correo electrónico: hendrawan@global.net.id
}

